\title{
DOZE ANOS DE DIAGNÓSTICO DE NEUROMICOSES EM UNIDADE ONCOLÓGICA: SÉRIE DE CASOS E REVISÃO SISTEMÁTICA
}

\author{
Twelve Years of Diagnosis of CNS Mycoses in Oncology Unit: Case Series and Systematic \\ Review
}

\author{
Monia Rieth Corrêa ${ }^{1}$ \\ Isabela Penha Martins de Araújo ${ }^{2}$ \\ Renata de Bastos Ascenço Soares ${ }^{3}$ \\ Cássia Silva de Miranda Godoy 45
}

Resumo: Objetivo: Elucidar o processo de diagnóstico cirúrgico de Neuromicoses e implicações prognósticas em uma unidade oncológica, entre os anos de 2009 e 2020. Métodos: Trata-se de um estudo retrospectivo em que foram analisados 129 laudos anatomopatológicos negativos para neoplasia, dos quais quatro casos concluíram etiologia fúngica, sendo três paracoccidioidomicose e um mucormicose. Uma revisão sistemática da literatura foi conduzida para referencial teórico, que levantou 77 casos para comparação. Resultados e Discussão: Os achados apresentados nesse estudo superaram a incidência mundial documentada de neuroparacoccidioidomicose em mais de 2 vezes. Nessa série, a mucormicose do sistema nervoso central refletiu apresentação típica de doença rinocerebral, com desfecho fatal. Todos os pacientes desse estudo eram HIV negativos, mas provenientes

\footnotetext{
1 Graduanda em Medicina, Pontifícia Universidade Católica de Goiás, Goiânia GO - Brasil. $₫$ monia.rc@hotmail.com (19 https://orcid.org/0000-0002-4874-1268.

2 Graduanda em Medicina, Pontifícia Universidade Católica de Goiás, Goiânia GO - Brasil. $₫$ isabelapmaraujo@gmail.com. (1) https://orcid.org/0000-0002-0915-4511.

${ }^{3}$ Professora Adjunta do Curso de Medicina, Escola de Ciências Médicas e da Vida, Pontifícia Universidade Católica de Goiás, Goiânia GO - Brasil. $₫$ renatasuarezbastos@gmail.com. 19 https://orcid.org/0000-00031029-325X.

${ }^{4}$ Professora Assistente do Curso de Medicina, Escola de Ciências Médicas e da Vida, Pontifícia Universidade Católica de Goiás, Goiânia GO - Brasil. $\varangle$ cassiamirandagodoy@ hotmail.com. 1 https://orcid.org/0000-00034893-0541

${ }^{5}$ Chefe do Controle de Infecção Hospitalar, Hospital Araújo Jorge, Associação de Combate ao Câncer em Goiás, Goiânia GO - Brasil. $₫$ cassiamirandagodoy@ hotmail.com. 1 https://orcid.org/0000-0003-4893-0541.
}

$\begin{array}{ccc}\text { Recebido em } & \text { Aceito em } & \text { Publicado em } \\ 22 / 07 / 2021 & 01 / 11 / 2021 & 09 / 11 / 2021\end{array}$


de áreas endêmicas do Centro-Oeste e Sudeste brasileiros, além de possuírem fatores de risco para infecções fúngicas invasivas e demonstrarem focos de acometimento extracraniano. Considerações finais: A ausência de investigação sobre o comprometimento em outros órgãos, bem como a espera por procedimento neurocirúrgico para o diagnóstico estão associadas a prejuízos à terapia adequada e tempo de hospitalização prolongada, que expõe os pacientes a maior risco de infecções nosocomiais. A subnotificação estatística supõe obstáculo importante ao avanço dos estudos em Neuromicoses, por isso é necessário investir em sistemas nacionais de vigilância e transformar as micoses endêmicas em doenças de notificação compulsória.

Palavras-chave: Neurocirurgia. Neuromicose. Mucormicose. Paracoccidioidomicose.

Abstract: Goal: To elucidate the process of surgical diagnosis of CNS mycosis and prognostic implications in an oncology unit, between years 2009 and 2020. Methodos: This is a retrospective study in which we analyzed 129 anatomopathological negative reports for neoplasia, resulting in four cases of fungal etiology, three Paracoccidioidomycosis and one Mucormycosis. A systematic review of the literature was also conducted for theoretical reference, which raised 77 cases for comparison. Results and Discussion: Our findings exceeded the documented incidence of Paracoccidioidomycosis by more than 2 times. In our series, Mucormycosis reflected a typical presentation of rhinocerebral disease, with fatal outcome. All our patients were HIV negative, but they came from endemic areas in the Midwest and Southeast of Brazil, had risk factors for invasive fungal infections and showed outbreaks of extracranial involvement. Final Considerations: The lack of investigation about the involvement in other organs, as well as waiting for a neurosurgical procedure for diagnosis are associated to damages to proper therapy and expanded hospitalization period, which exposes patients to greater risk for nosocomial infections. Statistical underreporting represents an important obstacle to the advancement of studies on CNS mycosis, and it is necessary to invest in national surveillance systems and transform endemic mycoses into compulsory reporting diseases.

Key words: Neurosurgery. Neuromycosis. Paracoccidioidomycosis. Mucormycosis.

\section{INTRODUÇÃO}

Apesar de relativamente raras, as Neuromicoses (NM), ou Infecções Fúngicas do Sistema Nervoso Central, se associam a elevadas taxas de morbiletalidade ${ }^{1,2}$. O incremento de sua incidência nas últimas décadas provavelmente se associa ao aumento da prevalência de condições imunossupressoras, resistência antifúngica, maior expectativa de vida, envelhecimento populacional ${ }^{2,3}$, mudanças geoclimáticas, crise socioambiental ${ }^{4,5}$, avanço das 
tecnologias diagnósticas e conscientização popular ${ }^{6}$. São condições que afetam tanto pessoas vivendo com HIV/AIDS (PVHA) quanto indivíduos HIV negativos, principalmente em sítios endêmicos. Diversas espécies endêmicas têm potencial neurotrópico, como o Cryptococcus gattii na Austrália, Papua Nova Guiné, México, Colúmbia Britânica, regiões africanas, sul da Califórnia e noroeste do Oceano Pacífico (EUA); Histoplasma spp. nos vales dos rios Ohio e Mississippi, nos Grandes Lagos e em regiões da América Latina; Paracoccidioides brasiliensis na Argentina, Colômbia, Venezuela, Equador, Paraguai e Brasil, além do México e porções da América central ${ }^{2,3,5-9}$; e Paracoccidioides lutzii predominantemente no CentroOeste brasileiro, Amazônia brasileira e Equador ${ }^{5}$.

Em nível mundial, a neurocriptococose é a infecção fúngica mais comum ${ }^{2,10,11}$, responsável por incidência anual de até 3,9 em 100.000 habitantes. A aspergilose invasiva tem incidência de 1 a 2 casos em 100.000 habitantes, dos quais 4 a 6\% cursam com envolvimento do SNC. Conquanto incomum, a mucormicose é altamente fatal e possui incidência de 0,04 a 0,17 por 100.000 habitantes, com $11 \%$ de acometimento do SNC e formação de abscessos em $9 \%^{2}$. Nos países endêmicos, a paracoccidioidomicose (PCM) conta com 1 a 3 novos casos anuais em 100.000 habitantes, dos quais 80\% são registrados no Brasil, onde a mortalidade pode chegar a 2,59 em um milhão. A maioria dos casos está nas regiões sul, sudeste e centrooeste, com dados recentes de 9,4 casos por 100 mil habitantes em Rondônia ${ }^{5,8,9,12}$. Séries de casos anteriores relatam prevalência de até $13,9 \%$ de neuroparacoccidioidomicose, número que pode chegar a $40 \%$ em estudos de imagem ${ }^{12}$. Apesar disso, inexistem, até o momento, informações epidemiológicas robustas, com grande possibilidade de subnotificação ${ }^{2,8}$.

As NM têm manejo desafiador, e a conduta cirúrgica é uma etapa quase sempre imprescindível. Contudo o diagnóstico por neurocirurgia supõe complicações graves e prognóstico reservado no pós-operatório. O "peso" dessas doenças é particularmente árduo onde há limitação de recursos e de disponibilidade para testes diagnósticos e terapias antifúngicas ${ }^{10}$. Este estudo apresenta quatro casos de NM diagnosticados por neurocirurgia em uma unidade oncológica de referência, num intervalo temporal de doze anos, discutindo o processo diagnóstico neurocirúrgico e implicações prognósticas, com foco para formas pseudotumorais e, ainda, à luz de evidências obtidas da literatura.

\section{MÉTODO}


Trata-se de um estudo retrospectivo de natureza aplicada e abordagem qualitativa, em que foram analisados, por meio da investigação de prontuários de pacientes submetidos a neurocirurgia, casos diagnosticados com Neuromicose entre os anos de 2009 e 2020, no Hospital Araújo Jorge (HAJ), unidade oncológica de Goiânia, Goiás.

A pesquisa teve início mediante aprovação do Comitê de Ética em Pesquisa do HAJ (Parecer n. ${ }^{\circ}: 4.302 .883$ ), segundo a Resolução 466/12 e complementares. A coleta de dados ocorreu de forma virtual, através do sistema interno do HAJ, para a pesquisa inicial dos laudos anatomopatológicos negativos para neoplasia, e física, na fase de análise dos prontuários completos. Foram excluídos aqueles referentes a infecções não fúngicas, e incluídos os de etiologia fúngica ou conclusão incerta. Ao todo foram realizadas 7866 neurocirurgias no período estudado, a partir das quais foram localizados 129 laudos negativos para neoplasias, 17 condizentes com neuroinfecções. Finalmente, foram encontrados quatro casos compatíveis com este estudo, discutidos adiante.

Para suporte teórico da discussão, transcorreu revisão sistemática de literatura científica nacional e internacional nas bases de dados PubMed, em que foram empregados os descritores “(CNS) AND (mycosis)”, Scielo e Periódicos CAPES, mediante os descritores "(Paracoccidioidomycosis) AND (CNS)". Foram incluídos relatos de casos e revisões realizados em seres humanos, publicados nos últimos 10 anos, nas línguas inglesa, portuguesa e espanhola, o que resultou em um total de 144 artigos. Destes foram selecionados 50 após a aplicação dos seguintes critérios de exclusão: falta de referências diretas no título ao sistema nervoso central ou seus componentes; falta de referências diretas no título a infecções fúngicas ou presença de referências a outras etiologias; relatos de casos em PVHA; relatos de casos em pacientes menores de 18 anos de idade; relatos de casos de apresentação não tumoral; e relatos de casos de infecções nosocomiais. Ao todo foram revisados 77 pacientes para comparação aos quatro pacientes deste estudo. Conforme os critérios do modelo PRISMA, as diferentes etapas da revisão podem ser observadas na Figura 1. 


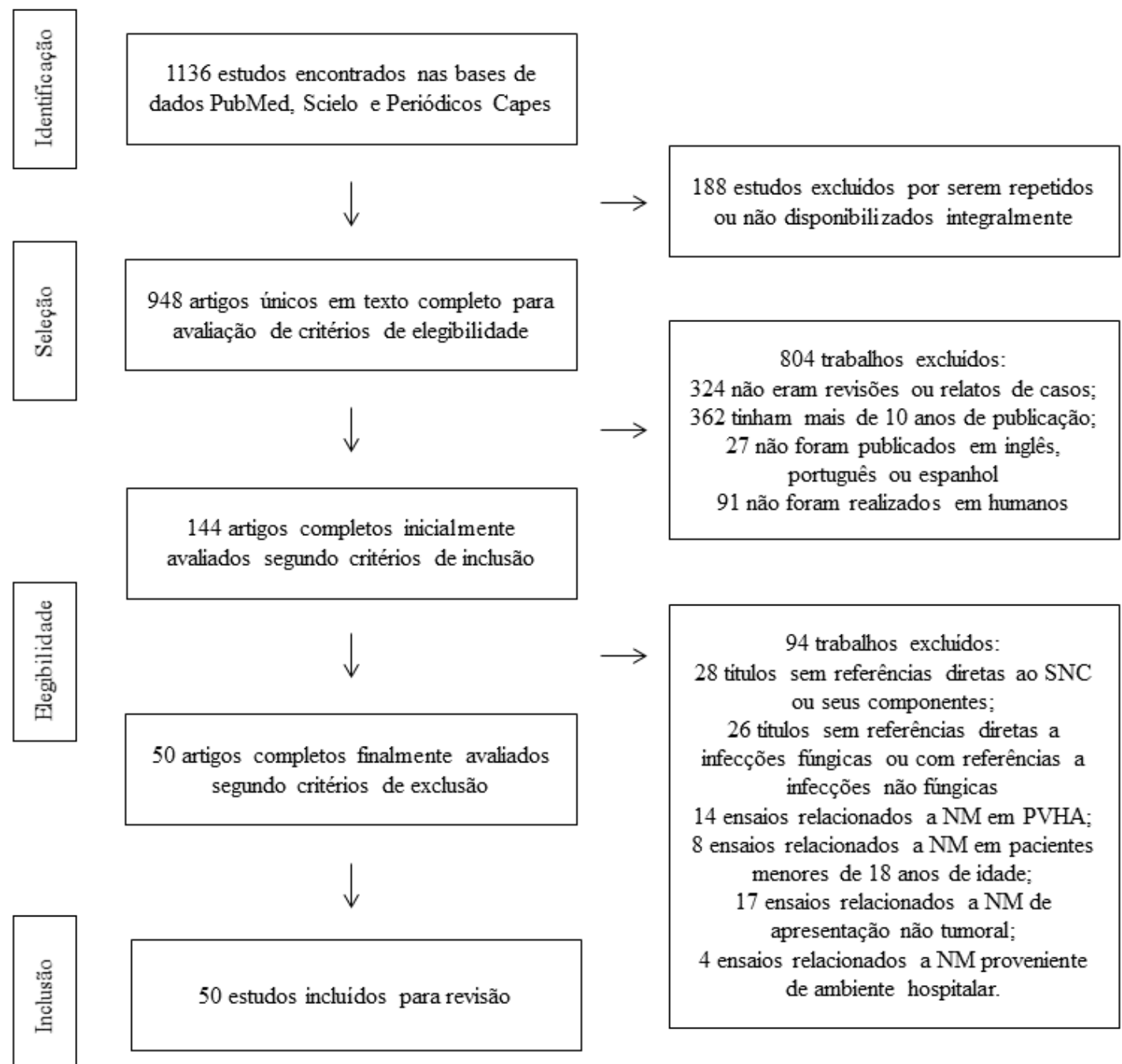

Figura 1. Fluxograma das etapas para revisão sistemática, conforme as recomendações PRISMA. Alguns estudos atendiam a mais de um critério de exclusão. Fonte: adaptado de "The PRISMA Group".

\section{CASOS}

Caso 1

Homem de 58 anos, HIV negativo, branco, lavrador, procedente de Inhumas, Goiás, foi admitido em 18 de abril de 2019 com quadro de hemiplegia esquerda e sinais de Síndrome de Hipertensão Intracraniana (SHIC). Tomografia computadorizada (TC) de crânio 
realizada 3 semanas antes noutro serviço, apresentava múltiplas lesões ovaladas hipodensas em região núcleo-capsular direita, a maior com cerca de 4,0 cm, e região subcortical cerebelar direita, de 2,6 cm, sugerindo implantes neoplásicos secundários. Também foi descrito, na fossa pituitária, lesão expansiva homogênea, levemente hiperatenuante $(1,7 \times 1,3 \mathrm{~cm})$, com extensão supra-selar e íntimo contato com o quiasma óptico, levantando a possibilidade de macroadenoma hipofisário. Nova TC de crânio realizada acrescentou indícios de desvio das estruturas da linha média para a esquerda, com possível herniação subfalcina e discreta hidrocefalia supra-tentorial.

No quarto dia de internação, foi submetido a ressecção cirúrgica macroscópica completa de lesão volumosa no subcaudado direito, que revelou lesão cística irregular, de cápsula endurecida e conteúdo necrótico, além de comunicação patente com o ventrículo lateral direito. O laudo anatomopatológico atestou presença de fungos arredondados de dupla membrana compatíveis com Paracoccidioides spp. Uma radiografia de tórax do mesmo dia demonstrou opacidades interstício-alveolares peri-hilares.

Após décimo-primeiro dia da admissão na UTI sob entubação orotraqueal, o paciente encontrava-se obnubilado, com risco de broncoaspiração, e foi submetido a Traqueostomia (TQT). Tomografia de tórax realizada doze dias após a neurocirurgia indicou consolidação e opacidades reticulares ramificadas nos lobos inferiores direito e esquerdo, respectivamente, associadas a opacificações em "vidro fosco" esparsas, assumido como novo processo inflamatório/infeccioso. No aspirado traqueal foi isolado Enterobacter cloacae multirresistente e o paciente fez uso de antibacterianos de largo espectro. Foi tratado com Anfotericina B de Complexo Lipídico (ABCL) por nove dias e, posteriormente, transferido ao Hospital de Doenças Tropicais (HDT), onde continuou o uso de ABCL por mais quatro semanas. Seguiu em tratamento ambulatorial no HDT com antifúngico oral (sulfametoxazol+trimetoprim) por 18 meses até a alta.

\section{Caso 2}

Homem de 66 anos, HIV negativo, pardo, carpinteiro, procedente de Jataí, Goiás, com história de alcoolismo e Doença de Chagas. Foi admitido em 02 de maio de 2016, com cefaleia progressiva, hemiparesia à esquerda e dificuldades na marcha havia um mês, bem como vômitos incoercíveis em jato, e regular estado geral. Tomografia computadorizada de 
crânio revelava hidrocefalia associada a múltiplas lesões em região parietal direita, com compressão de ventrículo lateral, e em região occipital, com captação intensa de contraste. Levantada suspeita de origem neoplásica, o paciente foi submetido, após cinco dias, a biópsia da lesão e Derivação Ventrículo-Peritoneal (DVP). Uma semana depois, o laudo anatomopatológico negou presença de elementos neoplásicos, porém resultou inconclusivo. Nove dias após a cirurgia, apresentava melhora importante do nível de consciência, sendo-lhe prescrito dexametasona ( $4 \mathrm{mg} 4 \mathrm{x} / \mathrm{dia}$ ).

Em consulta de retorno com infectologista, revelou-se diagnóstico antigo de PCM após biópsia de lesões orais em 2012, contudo abandonou tratamento com itraconazol e sulfametoxazol/trimetropim (SXT) ao terceiro mês. Evoluiu afebril, porém com piora clínica, diplopia, rigidez de nuca e persistência da SHIC pós obstrução da DVP, além de sonolência e períodos de confusão mental. No mês seguinte, foi submetido a nova biópsia e substituição da DVP, evoluiu com quadro gravíssimo, dispneico, taquicárdico, hipotenso, com roncos e estertores pulmonares. Iniciado uso de Anfotericina B Desoxicolato 50mg/dia, permaneceu por 48 dias em Unidade de Tratamento Intensivo (UTI), sob ventilação mecânica prolongada, e foi submetido a TQT após 9 dias de UTI. Novo resultado anatomopatológico demonstrou fungos leveduriformes com dupla camada e tamanho variável, consistente com Paracoccidioides spp. Durante a permanência na UTI foram identificadas três pneumonias associadas a ventilação mecânica por E. cloacae, Pseudomonas aeruginosa e Klebsiella pneumoniae multidrogarresistentes, motivo pelo qual faz uso de antibacterianos de largo espectro.

O paciente recebeu Anfotericina B Desoxicolato (AmBD) por 37 dias no HAJ e mais duas semanas após transferência para o HDT, onde continuou tratamento ambulatorial com SXT oral por dois anos, evoluindo com recuperação clínica apesar de persistência das lesões fibrocicatriciais pulmonares e sequela de lesão em verme cerebelar. Recebeu alta para cidade de origem.

\section{Caso 3}

Mulher de 75 anos, HIV negativa, parda, do lar, procedente de Vianápolis, Goiás, com história de Hipertensão Arterial Sistêmica e geofagia. Foi admitida em 16 de setembro de 2019 com suspeita de neoplasia maligna no nervo óptico esquerdo, paralisia facial central, 
dor, perda da acuidade visual à esquerda e emagrecimento (não quantificado) havia seis meses. A Ressonância Nuclear Magnética (RNM) de crânio da semana anterior apresentava sinais de sinusite e formação expansiva envolvendo nervo óptico esquerdo com extensão para fissura orbitária e sifão carotídeo esquerdos, alcançando espaço pterigoide esquerdo, apagando a gordura da parede posterior do seio maxilar ipsilateral e obliterando o Cavum de Meckel. A TC de crânio da admissão levantou suspeita de natureza neoplásica e acrescentou aumento volumétrico inespecífico e esclerose óssea na asa esfenoidal e paredes lateral e posterior da órbita esquerda. Em 18 de setembro, foi submetida a ressecção parcial da lesão, procedimento interrompido devido a infiltração de estruturas perilesionais. Em seguida, o laudo anatomopatológico revelou ausência de malignidade, porém com quadro inflamatório, focos de necrose, infiltrado leucocitário misto e agregados de leveduras e hifas compatíveis com agentes de mucormicose, fato confirmado pelo estudo imuno-histoquímico.

Ao segundo dia de pós-operatório recebeu alta precoce por exigência de familiares. Retornou apenas seis dias depois afebril, com rebaixamento do nível de consciência, sonolência, hematoma extenso em hemiface esquerda e secreção em região da ferida operatória. Nova TC de crânio evidenciou hematomas em regiões subdural frontotemporal e intraparenquimatosa frontotemporal esquerdas, com efeito compressivo sobre parênquima cerebral adjacente, obliteração parcial do ventrículo lateral esquerdo, apagamento de sulcos e fissuras cerebrais regionais, desvio contralateral das estruturas centromedianas e sinais de herniação subfalcina, além de ateromatose carotídea bilateral.

Utilizado AmBD (50 mg/dia) e Cefepima ( $2 \mathrm{~g}$ de 8/8h) via endovenosa por 13 dias, a paciente evoluiu gravemente torporosa, com gasping e insuficiência renal, pelo que foi transferida à UTI para assistência ventilatória, quando a AmBD foi substituída por ABCL (5 $\mathrm{mg} / \mathrm{kg} / \mathrm{dia}$ ) e a TC de crânio revelou hemossinusite esfenoidal à esquerda e calcificação de permeio compatível a colonização fúngica. Após entubação prolongada, a paciente foi submetida a TQT sem intercorrências. TC de tórax e abdome não indicavam achados específicos e a TC de crânio demonstrava expansão do edema para fossa pterigopalatina e espaço mastigador, danificando estruturas regionais, esclerose em assoalho de órbita lateral esquerda com extensão para cavidades paranasais e acentuação dos demais sulcos corticais e fissuras cerebrais. 
Nova TC de tórax realizada após uma semana evidenciou focos de consolidação pulmonar com pequenas cavitações no interior dos segmentos basais posteriores de ambos os lobos inferiores, com suspeita de processo pneumônico. O resultado de cultura com antibiograma de secreção traqueal revelou infecção bacteriana multirresistente $(K$. pneumoniae) e apesar da substituição dos antibacterianos, a paciente não apresentou melhora clínico-neurológica ou recuperação da função renal e progrediu para cuidados paliativos no $30^{\circ}$ dia de internação em UTI, evoluindo para óbito no $59^{\circ}$ dia do pós-operatório.

\section{Caso 4}

Homem de 60 anos, HIV negativo, branco, lavrador, procedente de Presidente Olegário, Minas Gerais, fumante, porém sem diagnósticos prévios. Foi admitido em 05 de outubro de 2020 com quadro progressivo de cefaleia e confusão mental, além de laudo de múltiplas lesões cerebrais com aspecto de metástase em RNM de crânio anterior. No dia 09 do mesmo mês, foi submetido a ressecção da lesão frontal à direita; e quatro dias depois, a outra ressecção, então de volumosa lesão cerebelar $(6 \mathrm{~cm}$, em seu maior diâmetro).

Recebeu alta ao $7^{\circ}$ dia do pós-operatório, contudo retornou à unidade após 11 dias, com confusão mental, febre intermitente, bem como tosse seca e dispneia. Em decorrência da pandemia por COVID-19, apesar do primeiro teste rápido negativo para detecção de anticorpos contra SARS-CoV-2, realizado em outra unidade, e como se apresentasse febril com infiltrado intersticial em "vidro fosco" à TC de tórax, um segundo exame de RT-PCR para o SARS-CoV-2foi realizado com resultado detectável.

O laudo anatomopatológico das biópsias foi localizado, evidenciando tecido extensamente necrótico com moderado infiltrado inflamatório linfo-histiocitário, reação granulomatosa e presença de estruturas leveduriformes grandes de membrana espessa birrefringente, sugestivas de Paracoccidioides spp. Nova TC havia identificado edema difuso do hemisfério cerebelar direito que determinava efeito compressivo sobre o quarto ventrículo e discreta hidrocefalia supratentorial, associados a formação expansiva subcortical temporal esquerda, isoatenuante e hipovascular, com $3,7 \times 4,1 \mathrm{~cm}$ em seus maiores eixos e halo de edema vasogênico. O paciente foi transferido para o Hospital das Clínicas, UFG, para tratamento da COVID-19, onde realizou terapia com ABCL durante quatro semanas, período 
após o qual recebeu alta em uso de SXT. Evoluiu com melhora clínica e segue em acompanhamento e tratamento regular previsto para 18 meses.

\section{RESULTADOS E DISCUSSÃO}

Tabela 1. Resumo e comparação das informações referentes aos casos de Neuromicoses diagnosticados por neurocirurgia no Hospital Araújo Jorge entre os anos de 2009 e 2020.

\begin{tabular}{|c|c|c|c|c|}
\hline Informação & Caso 1 & Caso 2 & Caso 3 & Caso 4 \\
\hline Idade (anos) & 58 & 66 & 75 & 60 \\
\hline Sexo & Masculino & Masculino & Feminino & Masculino \\
\hline Ocupação & Lavrador & Carpinteiro & Do lar & Lavrador \\
\hline $\begin{array}{l}\text { UF de } \\
\text { procedência }\end{array}$ & GO & GO & GO & MG \\
\hline Hábitos de vida & SI & Alcoolismo & Geofagia & Tabagismo \\
\hline $\begin{array}{l}\text { Infecção } \\
\text { fúngica prévia }\end{array}$ & Não & $\operatorname{Sim}(\mathrm{PCM})$ & Não & Não \\
\hline $\begin{array}{l}\text { Características } \\
\text { das lesões à TC }\end{array}$ & Hipodensidade & $\begin{array}{l}\text { Captação } \\
\text { intensa de } \\
\text { contraste }\end{array}$ & SI & $\begin{array}{l}\text { Isoatenuante } \\
\text { com halo de } \\
\text { edema } \\
\text { vasogênico }\end{array}$ \\
\hline 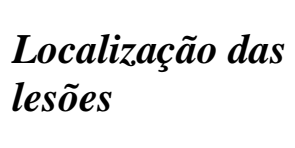 & $\begin{array}{l}\text { Nucleocapsular } \\
\text { D + cerebelo D } \\
\text { + fossa pituitária }\end{array}$ & $\begin{array}{l}\text { Região parietal } \\
\text { + cerebelo }\end{array}$ & $\begin{array}{l}\text { Órbita } \mathrm{E}+\text { seio } \\
\text { cavernoso E }\end{array}$ & $\begin{array}{l}\text { Lobo frontal + } \\
\text { cerebelo }\end{array}$ \\
\hline $\begin{array}{l}\text { Diagnóstico } \\
\text { histopatológico }\end{array}$ & $\begin{array}{l}\text { Paracoccidioido } \\
\text { micose }\end{array}$ & $\begin{array}{c}\text { Paracoccidioido } \\
\text { micose }\end{array}$ & Mucormicose & $\begin{array}{l}\text { Paracoccidioid } \\
\text { omicose }\end{array}$ \\
\hline $\begin{array}{l}\text { Intervenções } \\
\text { terapêuticas }\end{array}$ & $\begin{array}{l}\text { ABCL + } \\
\text { conduta } \\
\text { cirúrgica }\end{array}$ & $\begin{array}{l}\text { AmBD + } \\
\text { conduta } \\
\text { cirúrgica }\end{array}$ & $\begin{array}{c}\text { AmBD/ABCL + } \\
\text { conduta } \\
\text { cirúrgica }\end{array}$ & $\begin{array}{c}\text { ABCL + } \\
\text { conduta } \\
\text { cirúrgica }\end{array}$ \\
\hline Desfecho & Alta & Alta & Óbito & $\begin{array}{c}\text { Em } \\
\text { seguimento }\end{array}$ \\
\hline
\end{tabular}

SI: sem informações. D: direito. E: esquerdo. ABCL: Anfotericina B de Complexo Lipídico; AmBD: Anfotericina B Desoxicolato. SXT: Sulfametoxazol/trimetoprim.

A Tabela 1 reúne um resumo dos quatro casos encontrados neste estudo. Dentre eles, três correspondiam ao sexo masculino, e um ao feminino. Essa predominância também foi observada entre os 77 casos revisados, porém numa proporção menor $(49,3 \%$ homens e 35,1\% mulheres). Em 15,6\% dos casos revisados, o parâmetro sexo foi ignorado. À admissão, apenas um paciente não foi considerado idoso (58 anos), já os outros três tinham pelo menos 60 anos. Dos 77 casos revisados, apenas 15,6\% correspondiam a indivíduos acima dos 60 anos, enquanto a faixa etária de 50 a 59 revelou-se a de maior incidência (35\%). A explicação para a diferença na idade pode estar na cronicidade das formas peseudotumorais de PMC do 
SNC, em menor número na literatura revisada, mas maioria na série analisada. A distribuição por idade dos casos revisados pode ser verificada no Gráfico 1.

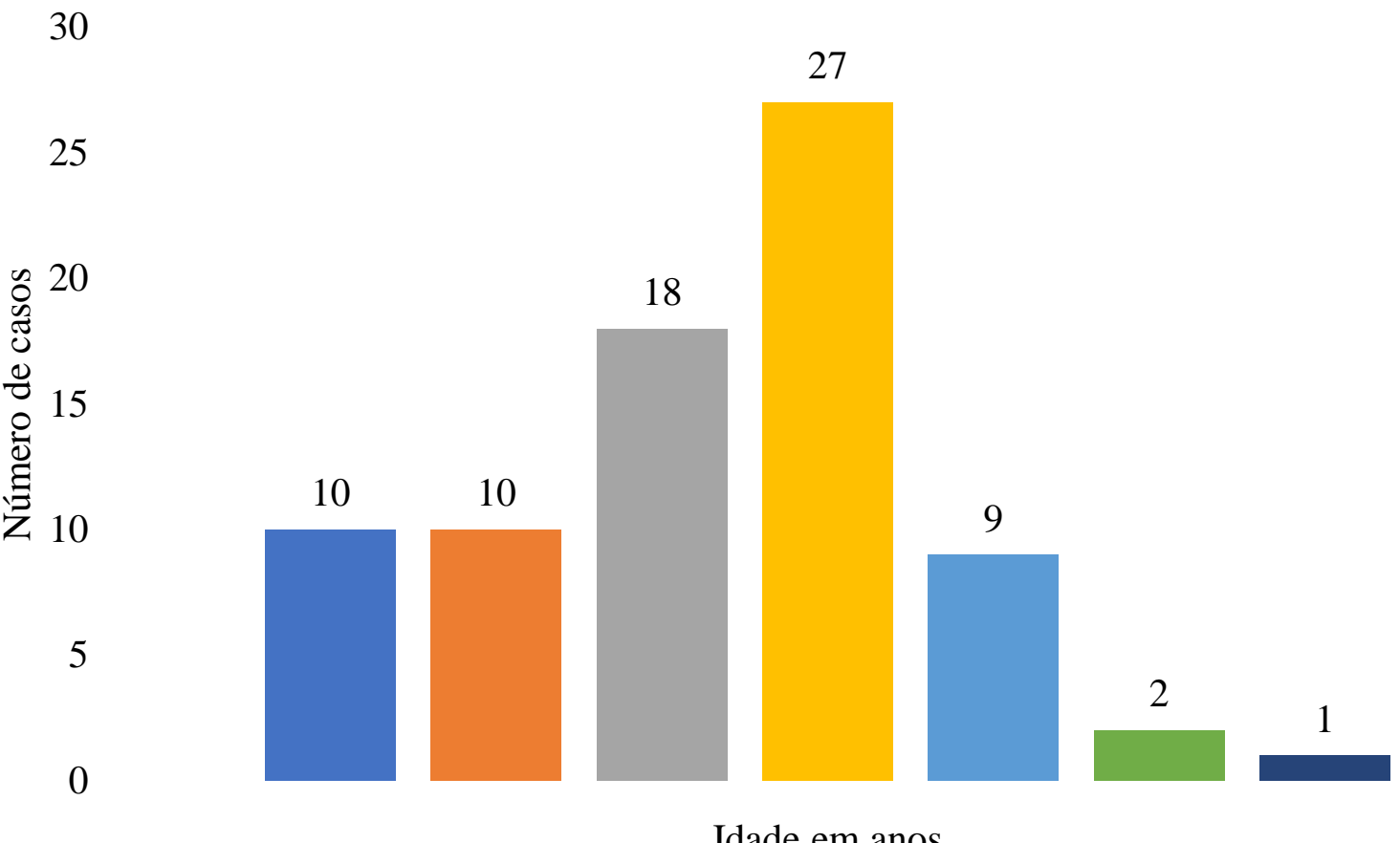

$\square 18$ a $29 \square 30$ a $39 \square 40$ a $49 \square 50$ a $59 \square 60$ a $69 \square 70$ a $79 \square 80$ ou mais

Gráfico 1. Distribuição de 77 casos de Neuromicoses relatados entre 2011 e 2021, segundo a idade em anos.

Após quase 8.000 neurocirurgias realizadas em 12 anos no $\mathrm{HAJ}$, foram encontrados quatro casos de NM pseudotumoral diagnosticados por neurocirurgia em pacientes HIV negativos com 18 anos ou mais (uma mucormicose e três PCM). Essa amostra supera em mais de 2 vezes a incidência mundial documentada de $\mathrm{PCM}^{5}$ em regiões endêmicas e vai ao encontro da literatura revisada, em que foram encontrados 13 casos $(16,9 \%)$ correspondentes em dois estudos latino-americanos; 12 deles relatados no Brasil ${ }^{12}$. Os únicos casos identificados em regiões não endêmicas não puderam ser incluídos nesta revisão por se tratarem de PVHA ou formas meningoencefálicas de NM. As mucormicoses apareceram em 9,1\% dos casos revisados. O Gráfico 2 exibe a distribuição destes quanto ao diagnóstico histopatológico. 


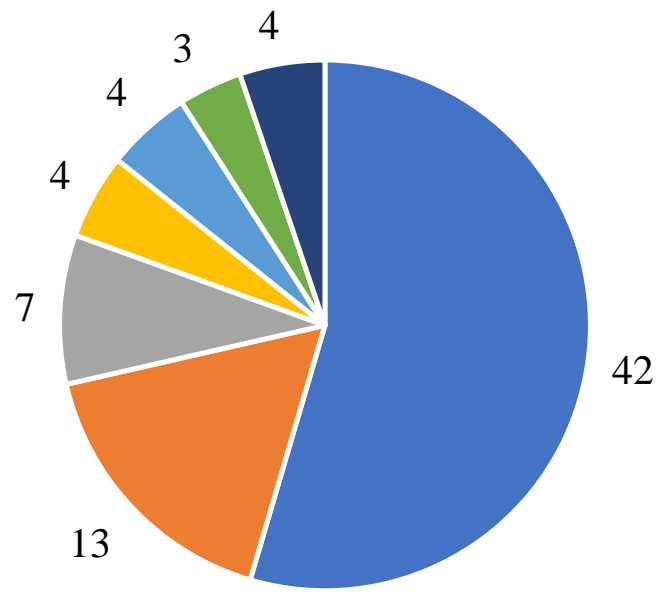

- Aspergillus spp. $\quad \square$ Paraccocidioides spp. $\quad$ Mucormicoses
$\square$ Criptococcus spp. $\quad \square$ Feo-hifomicoses $\quad \square$ Scedosporium spp.
- Outros

Gráfico 2. Distribuição de 77 casos de Neuromicoses relatados entre 2011 e 2021, segundo o diagnóstico histopatológico. Outros: Curvularia spp., Fusarium spp., Histoplasma spp. e basidiomicoses.

As formas pseudotumorais incluem abscessos, granulomas, nódulos, cistos, e lesões vasculares, como vasculites, aneurismas e infartos ${ }^{4}$. Interrupções por trauma e outros estados que aumentam a permeabilidade da barreira hematoencefálica (BHE) facilitam a invasão, seja por disseminação hematogênica, linfática ou por contiguidade a infecções dos seios da face ${ }^{6,7,13}$. Na maioria dos acometimentos pseudotumorais, a disseminação decorre de infecção primária das vias aéreas após inalação de conidiose aerotransportada, como na PCM, aspergilose, feo-hifomicose, criptococose e histoplasmose ${ }^{2,5,7,8,14-17}$. Essas apresentações podem levar a efeitos de massa importantes e edema cerebral com SHIC. Em pacientes HIV negativos, a neovascularização decorrente da reação granulomatosa produz captação de contraste semelhante à de tumores do $\mathrm{SNC}^{14}$.

A PCM, causada sobretudo por P. brasiliensis e P. lutzii, é considerada a infecção fúngica mais importante na América Latina ${ }^{5,8}$. Em geral, a neuroinfecção é ocasionada pela disseminação proveniente de focos pulmonares e representa alto potencial incapacitante, principalmente para indivíduos cronicamente expostos, como os trabalhadores rurais, além de associações a tabagismo e alcoolismo ${ }^{5,9}$. Tipicamente relatadas na forma crônica, as lesões do 
SNC podem produzir granulomas e abscessos, com cefaleia, déficit motor, convulsões, sinais cerebelares, hidrocefalia e alterações comportamentais e do nível de consciência, simulando lesões neoplásicas da fossa posterior ${ }^{5,9}$. A forma pseudotumoral, mais comum, demanda tratamento cirúrgico excisional e uso de $\mathrm{AmBD}^{5}$.

Nesse estudo, assim como nos 13 casos revisados, todos os pacientes diagnosticados com PCM eram provenientes de regiões endêmicas (Centro-Oeste e Sudeste), e suas ocupações (dois lavradores e um carpinteiro) lhes conferiam exposição prolongada aos esporos de Paracoccidioides spp. Também em consonância às evidências encontradas, todos apresentaram lesões em fossa posterior, entre outras localizações, bem como comprometimento pulmonar. Além disso, um paciente referiu história de alcoolismo e outro, tabagismo. SHIC e comprometimento da consciência compuseram o quadro em todos os casos dessa série, e déficit motor e cefaleia, em dois casos, sintomas também compatíveis à literatura revisada. Um paciente apresentava diagnóstico anterior de PCM em cavidade oral, dado somente registrado após o procedimento cirúrgico, o que demonstra a importância de uma história completa para avaliação de diagnósticos diferenciais e da adesão ao tratamento preconizado.

As mucormicoses, causadas por fungos da ordem Mucorales, (Rhizopus, Mucor e outros), invadem o parênquima cerebral via hematogênica a partir de infecções de vias aéreas, invasão sinocraniana ou doença cutânea ${ }^{2-4,6,8,14}$. Acometem principalmente os lobos frontal e temporal, e estão associadas a altas taxas de mortalidade, principalmente com diagnóstico tardio. Não raramente, a invasão por contiguidade proveniente dos seios da face envolve todas as estruturas ao longo do seu caminho e pode se estender aos seios cavernosos ${ }^{16}$. Evidências apontam para associação com destruição óssea tardia ${ }^{1,14,16}$. Abscessos cerebrais solitários ou múltiplos podem estar associados a episódios de afogamento ou trauma e sinais neurológicos focais $^{1,4,16}$.

Como pode ser observado na Figura 2, paciente do sexo feminino (caso 3) apresentava lesões em região frontotemporal, com apresentação típica de doença rinocerebral, sinais de infecção dos seios da face, com acometimento de todas as estruturas no caminho invasivo até o SNC, e degeneração óssea. De notável semelhança a evidências na literatura, essa história aponta para envolvimento avançado do SNC desde a admissão, o que já configura particular desafio frente uma NM com alta taxa de mortalidade não obstante 
intervenção adequada. Atrasos na dispensação de ABCL via Divisão de Micoses do Ministério da Saúde e a toxicidade renal provocada pela AmBD resultaram em pior prognóstico. Apesar de não ter sido relatado diagnóstico prévio de diabetes, fator de risco mais associado à mucormicose ${ }^{3}$, a paciente apresentava história de geofagia, o que supõe maior exposição a conídios presentes no solo e reforça a importância da investigação criteriosa dos fatores de risco.

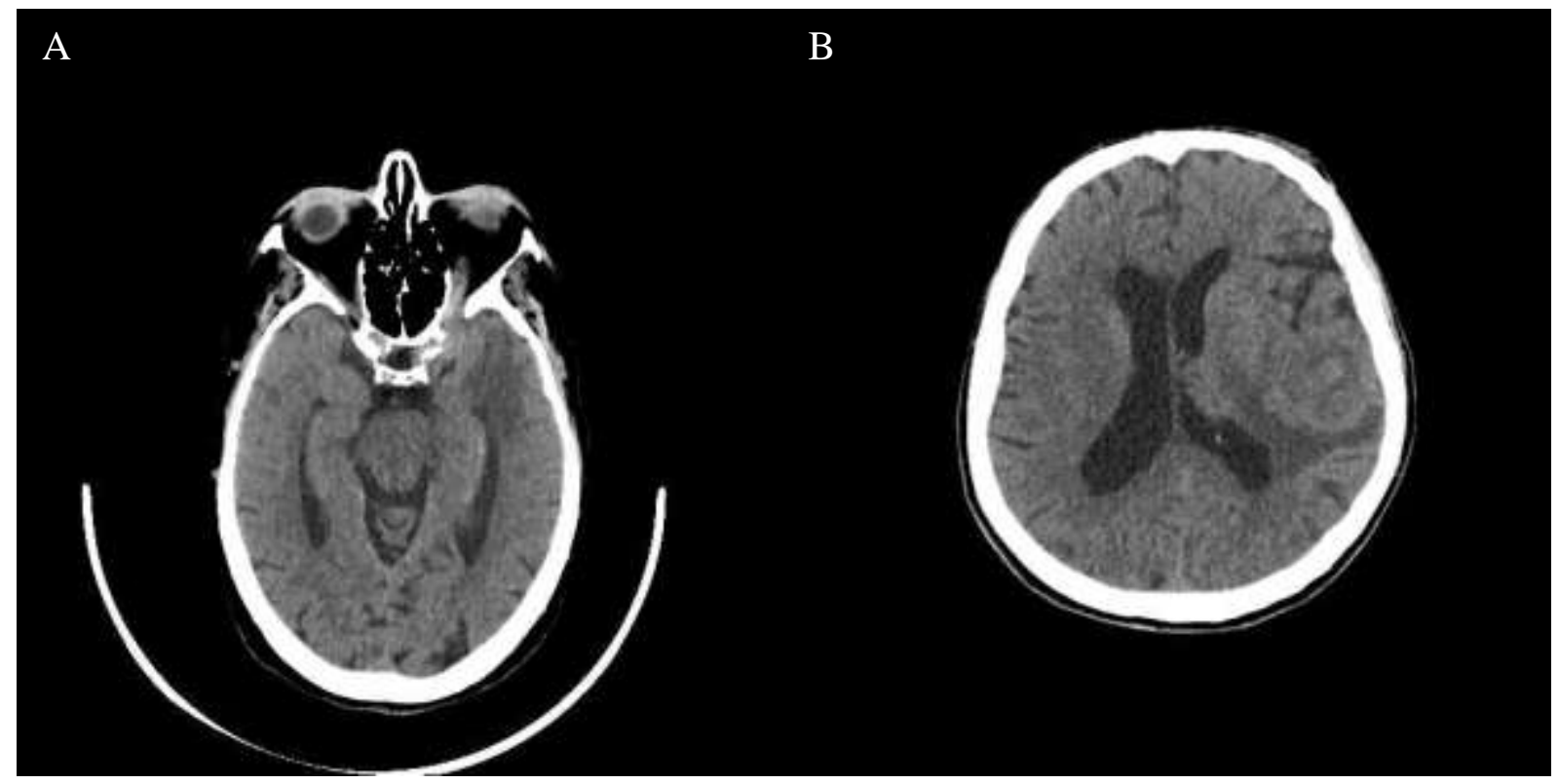

Figura 2. Tomografias computadorizadas de crânio. A) lesão expansiva em fossa craniana média esquerda com extensão ao ápice orbitário e nervo óptico ipsilaterais, envolvendo o sifão carotídeo esquerdo e alcançando o polo inferior do parênquima temporal com realce importante pelo contraste, em paciente com quadro de dor, paralisia facial central, prejuízo da acuidade visual à esquerda, emagrecimento e sinais de sinusite em ressonância nuclear magnética anterior (caso 3). B) formação expansiva subcortical temporal esquerda, isoatenuante e hipovascular, medindo $3,7 \times 4,1 \mathrm{~cm}$ nos seus maiores eixos axiais, associada a halo de edema vasogênico, em paciente com quadro progressivo de cefaleia e confusão mental previamente submetido à ressecção de lesão frontal à direita (caso 4).

Apesar de ausentes nessa série, criptococose, feo-hifomicoses e outras (basidiomicoses, histoplasmose e neuroinfecções por Curvularia spp.) apareceram em 5,2\% dos casos revisados cada. Em indivíduos não HIV essas etiologias foram associadas a lesões pseudotumorais que geralmente cursam com SHIC, cefaleia intensa, alterações do nível de consciência, prejuízos sensoriais (visão) e motores, com comprometimento de nervos cranianos $^{2-4,7,8,11,14-16}$. Distúrbios de memória e personalidade foram relatados em NM por 
Criptococcus spp. e Histoplasma spp. Febre está presente em até 98\% dos pacientes com histoplasmose do $\mathrm{SNC}^{2,8,11,14}$. Estima-se que o Brasil seja o terceiro maior responsável $(4,2 \%)$ por casos de NM por Cladophialophora bantiana, atrás apenas da Índia (32,5\%) e dos Estados Unidos $(24,2 \%)^{17}$. A aspergilose do SNC, com destaque para A. fumigatus, tampouco apareceu nesse estudo, mas foi responsável por 54,5\% dos casos revisados. Suas manifestações pseudotumorais iniciam-se tipicamente com infarto seguido por formação de granulomas ou abscessos, com febre, alteração do nível de consciência, cefaleia, sinais neurológicos focais, hemiparesia, convulsões, e embolia séptica ${ }^{1,3,4,8,10,16}$.

Os sinais e sintomas levantados nessa série podem ser observados na Tabela 2 . Todos os pacientes desse estudo apresentaram SHIC e efeito de massa, também frequentes na literatura (41,5 e 44,1\%, respectivamente). O rebaixamento do nível de consciência esteve presente também em todos os casos, porém com frequência relativamente menor segundo outros autores $(31,2 \%)$. No estudo em questão, todos cursaram com sinais de infecção extracraniana (focos pulmonares nos pacientes de PCM e dos seios da face na paciente de mucormicose), relato frequente também em outros trabalhos (46,7\%). Presente em três casos dessa série, a cefaleia e o prejuízo motor apareceram em aproximadamente metade (48 e 49,3\%, respectivamente) dos casos revisados. Prejuízo sensorial foi relatado uma vez nessa série, mas esteve em 35,1\% dos casos da literatura. Apesar de relatada com frequência de aproximadamente 40,2\%, febre relacionada a NM foi ausente nesse estudo. Síndrome consumptiva foi suspeitada uma vez nessa série e em $16,9 \%$ da literatura revisada.

Tabela 2. Comparação de sinais e sintomas entre os casos de Neuromicoses diagnosticados por neurocirurgia no Hospital Araújo Jorge entre os anos de 2009 e 2020.

\begin{tabular}{lcccc}
\hline Sinais e sintomas & Caso 1 & Caso 2 & Caso 3 & Caso 4 \\
\hline Sinais de Hipertensão Intracraniana & Sim & Sim & Sim & Sim \\
Efeito de massa & Sim & Sim & Sim & Sim \\
Cefaleia progressiva & Não & Sim & Sim & Sim \\
Rebaixamento do nível de consciência & Sim & Sim & Sim & Sim \\
Prejuízo motor & Sim & Sim & Sim & Não \\
Prejuízo sensorial & Não & Não & Sim & Não \\
Febre & Não & Não & Não & Não* \\
Sinais de Síndrome Consumptiva & Não & Não & Sim** & Não \\
Sinais de focos infecciosos extracranianos & Sim & Sim & Sim & Sim \\
\hline *O caso 4 apresentou febre, porém relacionados a quadro de COVID-19, não de NM. **No \\
caso 3, não pode ser confirmada síndrome consumptiva.
\end{tabular}


Outras manifestações encontradas na revisão, como prejuízo cognitivo, sintomas psiquiátricos, convulsões e dor ocular, não foram apresentadas pelos pacientes dessa série (18-50). A literatura revisada revelou $31,2 \%$ de óbitos, enquanto nessa série houve apenas um. Essas e outras informações podem ser encontrados na Tabela 3.

Tabela 3. Frequência de informações obtidas a partir de revisão sistemática de 77 casos de Neuromicoses relatados por outros autores entre 2011 e 2021.

\begin{tabular}{lc}
\hline Sexo & Total \\
\hline Feminino & $35,1 \%(27)$ \\
Masculino & $49,3 \%(38)$ \\
Ignorado & $15,6 \%(12)$ \\
\hline Sinais $\boldsymbol{e}$ sintomas & Total \\
\hline Sinais de Hipertensão Intracraniana & $41,5 \%(32)$ \\
Efeito de massa & $44,1 \%(34)$ \\
Cefaleia & $48 \%(37)$ \\
Dor (exceto cefaleia) & $6,5 \%(5)$ \\
Rebaixamento do nível de consciência & $31,2 \%(24)$ \\
Prejuízo motor & $49,3 \%(38)$ \\
Prejuízo sensorial & $35,1 \%(27)$ \\
Prejuízo cognitivo & $7,8 \%(6)$ \\
Sintomas psiquiátricos & $3,9 \%(3)$ \\
Febre & $40,2 \%(31)$ \\
Convulsões & $26 \%(20)$ \\
Sinais de Síndrome Consumptiva & $16,9 \%(13)$ \\
Sinais de focos infecciosos extracranianos & $46,7 \%(36)$ \\
\hline Desfechos $\boldsymbol{e}$ complicações & Total \\
\hline Melhora clínica & $55,8 \%(43)$ \\
Piora* & $2,6 \%(2)$ \\
Óbito & $31,2 \%(24)$ \\
Sequela neurológica & $13 \%(10)$ \\
Infecção bacteriana nosocomial & $7,8 \%(6)$ \\
Ignorado & $10,4 \%(8)$ \\
\hline Seguta &
\end{tabular}

*Seguem em acompanhamento

Nessa série, um paciente não declarou comorbidades, enquanto outros dois apresentavam diagnósticos prévios crônicos: um com história pregressa de Doença de Chagas, e outro em tratamento para Hipertensão Arterial Sistêmica. Não foi possível atestar sobre diagnósticos prévios ou hábitos de vida de um paciente por falta dessas informações no prontuário. Nenhum dos pacientes desse estudo era HIV positivo, mas todos apresentavam fatores de risco para NM e foram submetidos a procedimento neurocirúrgico e biópsia para esclarecimento de suspeita neoplásica. 
A RNM e a TC, embora comumente negativas e pouco específicas, podem fornecer características morfológicas interessantes, além de desempenhar papéis auxiliares na definição de diagnósticos diferenciais, da região anatômica afetada e de fontes extras de infecção. Em abscessos fúngicos, a difusão reduzida é precoce devido à alta viscosidade de seu conteúdo. Ao contrário dos abscessos bacterianos, o padrão fúngico na RNM é frequentemente heterogêneo, semelhante a um anel periférico, ou em padrão pontilhado nas lesões menores. O estado imunológico intacto leva à formação de realces robustos, mais raro em pacientes HIV positivos. Em T2, Aspergillus spp. possui aspecto semelhante a um alvo, com intensidade periférica intermediária ou baixa e hiperintensidade central ${ }^{3,14}$. Criptococomas têm aparência patognomônica à RNM: hipointensidade em T1 e centro hiperintenso cercado por anel hipointenso em T2, imitando tumor cerebral necrótico ${ }^{3,6,14}$. Já os histoplasmomas são hipointensos em $\mathrm{T} 1$ e variáveis em $\mathrm{T} 2$, com realce do anel periférico $^{2,3,14}$. Na PCM, as lesões intraparenquimatosas podem ser totalmente sólidas, de padrão granulomatoso compacto, ou ter área central de necrose, semelhante a abscessos bacterianos. São encontradas lesões irregulares, com efeito de massa e realce periférico em anel após contraste em $76 \%$ delas, bem como edema perilesional em $82 \%{ }^{9}$. Nesse estudo, as características tomográficas das lesões por PCM demonstraram hipodensidade (caso 1), captação intensa de contraste (caso 2) e halo de edema vasogênico (caso 4), como demonstra a Figura 2.

Não obstante, o padrão ouro para diagnóstico definitivo requer biópsia de amostras da área suspeita seguida de cultura e avaliação histopatológica direta. As biópsias estereotáxicas guiadas por imagem, em especial, conferem alto índice diagnóstico ${ }^{4}$. Ao exame histopatológico, Aspergillus spp. apresentam hifas septadas com ramificações dicotômicas, enquanto Mucorales spp. são mais irregulares, não septadas ou pauci-septadas, ramificadas em ângulos retos. As leveduras de $H$. capsulatum são pequenas, em brotamento; enquanto as de $P$. brasiliensis são grandes, com leveduras filhas em configuração de "roda de leme",2,8,14,16.

As técnicas baseadas em branqueadores ópticos (Calcofluor, Blankophor) possuem altas sensibilidade e especificidade ${ }^{7}$. O método da tinta nanquim é altamente específico para criptococose $(>90 \%)$, porém pouco sensível ${ }^{4}$. Para diagnóstico de feohifomicoses, deve-se utilizar coloração específica (Fontana-Mason), já que as hifas podem 
não parecer pigmentadas a princípio ${ }^{17}$. Os preparados com hematoxilina e eosina (prata Gomori metenamina, ácido periódico Schiff) também constituem técnicas de coloração de grande importância ${ }^{7,8,16}$. Em geral, a análise do líquido cefalorraquidiano (LCR) demonstra hipoglicorraquia, hiperproteinorraquia e altos níveis de IgG. A hipocelularidade é sinal de mau prognóstico ${ }^{1}$. A pleocitose linfocítica frequentemente se associa ao $C$. neoformans; e predominância neutrofílica, a Aspergillus spp. ${ }^{7}$.

A pesquisa de anticorpos possui sensibilidade entre 38 e $92 \%$, e a detecção de componentes da parede celular ( $\beta$-glucanos, galactomanano, manano, quitina), entre 64 e $90 \%{ }^{1,2,4,6-8,16}$. A utilização da Reação em Cadeia da Polimerase é a técnica que apresenta maior precisão, com sensibilidade e especificidade que chegam a 100 e 93\%, respectivamente, para amostras de LCR. De forma semelhante, a hibridização por fluorescência in situ apresenta potencial para tornar-se o novo padrão ouro em $\mathrm{NM}^{7,8}$.

Mesmo após cultura negativa, a análise histopatológica positiva para infecção fúngica, é considerada prova diagnóstica suficiente, mas requer biópsia cerebral ${ }^{8}$. Entretanto, em muitos casos, isso não é possível devido a riscos associados ao procedimento, principalmente para pacientes gravemente enfermos, além de atrasar significativamente o diagnóstico e o tratamento ${ }^{4}$, o que pode contribuir para desfechos negativos. O diagnóstico de NM por neurocirurgia também implica em internação por tempo prolongado, expondo a maior risco de infecções nosocomiais, como aconteceu a três dos pacientes dessa série e a $7,8 \%$ dos casos revisados.

A escolha da medicação antifúngica depende do seu espectro de atuação, ações fungistática e fungicida, e características farmacocinéticas e farmacodinâmicas no SNC, como a penetração dos medicamentos na $\mathrm{BHE}^{18}$. Considerada terapia primária para aspergilose do SNC, o voriconazol tem ótimas penetração pela BHE e concentrações em abscessos cerebrais $^{1,4,6-8,10,16,18}$, além de apresentar ação contra $P$. brasiliensis ${ }^{12}$; enquanto as AmB, de menor penetração, é normalmente reservada para pacientes intolerantes ou refratários, sendo as formulações lipídicas mais bem toleradas e recomendadas como primeira linha para mucormicose e fusariose $\mathrm{e}^{4,6,16,18}$. Combinada à $\mathrm{AmB}$, a flucitosina exibe atividade contra Cryptococcus spp. e Aspergillus spp, porém não é recomendada em monoterapia, pelo risco de resistência. O posaconazol, também de fraca penetração no SNC, pode ser indicado em segunda linha para mucormicose e como terapia de resgate na aspergilose e histoplasmose ${ }^{6,18}$, 
para as quais isavuconazol também mostra atividade satisfatória ${ }^{8,10,18}$. As equinocandinas são consideradas adjuvantes para aspergilose ${ }^{6,18}$. Até o momento, não existe terapia medicamentosa comprovadamente eficaz para $C$. bantiana $^{17}$. Para outros fungos não suscetíveis a agentes de boa penetração pode-se utilizar $\mathrm{AmB}^{8}$. A combinação sulfametoxazol/trimetoprim tem se mostrado eficaz contra a PCM, com boa penetração pela $\mathrm{BHE}^{8}$. Novos agentes estão em investigação, com destaque para o potencial promissor do F901318 (olorofim), que tem se demonstrado ativo contra Aspergillus spp., H. capsulatum, Fusarium spp. e outros ${ }^{18}$.

Apresentações clínicas com complicações ameaçadoras, como lesões expansivas, SHIC ou hemorragia, exigem intervenção neurocirúrgica. Os critérios propostos na literatura incluem hidrocefalia, casos refratários à terapia antifúngica, efeitos de massa, hemorragia subaracnóidea, deterioração neurológica, declínio da visão, proptose progressiva e diagnóstico inconclusivo $^{8}$, em casos de lesões corticais ou subcorticais superficiais, como abscessos e granulomas, em áreas não eloquentes do cérebro. Granulomas e abscessos intramedulares podem ser tratados em primeira linha com drenagem ou excisão ${ }^{4}$. Nas neuromucormicoses, a melhor estratégia parece ser a abordagem cirúrgica agressiva associada a antifungicoterapia precoce com ABCL e posteriormente posaconazol ou isavuconazol ${ }^{2,6,14}$. Aspergilomas intracranianos são majoritariamente tratados com ressecção cirúrgica seguida de AmB convencional, porém com grande índice de mortalidade e resultados que não superam o uso de itraconazol ${ }^{6}$. O tratamento à base de sulfonamida, AmB e intervenção cirúrgica são as abordagens mais relatadas para PCM do SNC, porém com 44,1\% de mortalidade geral ${ }^{12}$.

\section{CONCLUSÕES}

Sem terapia eficaz, as NM são uniformemente fatais. As lesões pseudotumorais do SNC causadas por fungos podem assemelhar malignidade e induzir a erros diagnósticos que resultam em morbidade desnecessária. A ausência de investigação sobre o comprometimento em outros órgãos, como pulmões e cavidade oral, bem como a espera por procedimento neurocirúrgico para o diagnóstico (biópsia) estão associadas a prejuízos à terapia em tempo oportuno e período de hospitalização prolongado, que expõe os pacientes a maior risco de infecções nosocomiais. Na maioria das vezes, a combinação de medicamentos antifúngicos e conduta cirúrgica aumenta a taxa de sobrevivência e está associada a melhores 
prognósticos. Entretanto a intervenção cirúrgica, quando não associada a suspeição de etiologia fúngica entre os diagnósticos diferenciais, pode representar um fator de pior prognóstico.

$\mathrm{O}$ achado expressivo de PCM na amostra analisada superou a incidência mundial documentada, evidenciando a importância da epidemiologia para a consideração de diagnósticos diferenciais em lesões pseudotumorais do SNC. A subnotificação estatística supõe obstáculo importante ao avanço dos estudos em NM, no meio profissional e acadêmico, mas pode ser minimizada ao se investir em sistemas nacionais de vigilância e ao se transformar as micoses endêmicas em doenças de notificação compulsória.

Neste estudo podemos destacar algumas limitações. A composição da amostra, em função da pesquisa ter sido conduzida em um centro oncológico, todos os casos analisados corresponderam às apresentações pseudotumorais das Neuromicoses. Devido à situação de pandemia pela COVID-19, não foi possível incluir outros centros. Além disso, os casos foram obtidos mediante o resultado anatomopatológico das lesões ressecadas ou biopsiadas, sem que houvesse cultura para uma definição etiológica mais precisa. Outra restrição encontrada diz respeito à reduzida disponibilidade de tecnologia diagnóstica na unidade estudada, como a utilização de PCR ou marcadores teciduais específicos.

\section{REFERÊNCIAS}

1 PANACKAL, A. A.; WILLIAMSON, P. R. Fungal Infections of the Central Nervous System. Continuum (Minneap Minn), v. 21, n. 6, p. 1662-1678, 2015.

2 JIA, D. T.; THAKUR, K. Fungal Infections of the Central Nervous System. Semin Neurol, v. 39, n. 3, p. 343-347, 2019.

3 MATHUR, M.; JOHNSON, C. E.; SZE, G. Fungal Infections of the Central Nervous System. Neuroimaging Clin N Am, v. 22, n. 4, p. 609-632, 2012.

4 KOURBETI, I. S.; MYLONAKIS, E. Fungal central nervous system infections: Prevalence and diagnosis. Expert Rev Anti Infect Ther, v. 12, n. 2, p. 265-273, 2014.

5 SHIKANAI-YASUDA, M. A. et al. II Consenso Brasileiro em Paracoccidioidomicose - 2017. [s.l: s.n.]. v. 27

6 MURTHY, J. M. K.; SUNDARAM, C. Fungal infections of the central nervous 
system. In: Handb Clin Neurol. 1. ed. [s.1.] Elsevier B.V., 2014. v. 121p. 1383-1401.

7 GÓRALSKA, K.; BLASZKOWSKA, J.; DZIKOWIEC, M. Neuroinfections caused by fungi. Infection, v. 46, n. 4, p. 443-459, 2018.

8 SCHWARTZ, S. et al. Advances in the diagnosis and treatment of fungal infections of the CNS. Lancet Neurol, v. 17, n. 4, p. 362-372, 2018.

9 ROSA JÚNIOR, M. et al. Imaging paracoccidioidomycosis: A pictorial review from head to toe. Eur J Radiol, v. 103, p. 147-162, 2018.

10 BLOCH, K. C.; BAILIN, S. S. Update on fungal infections of the central nervous system: Emerging pathogens and emerging diagnostics. Curr Opin Infect Dis, v. 32, n. 3, p. 277-284, 2019.

11 COLOMBO, A. C.; RODRIGUES, M. L. Fungal colonization of the brain: anatomopathological aspects of neurological cryptococcosis epidemic in HIV patients ( Armstrong-James et al . people die each year because of systemic fungal cryptococcosis presented in the last ( 9 th ) edition and Cryp. An Acad Bras Cienc, v. 87, n. 2 Suppl, p. 1293-1309, 2015.

12 FRANCESCONI, F. et al. Long-term outcome of neuroparacoccidioidomycosis treatment Resultado de longo prazo no tratamento da neuroparacoccidioidomicose. Rev Soc Bras Med Trop, v. 44, n. 1, p. 22-25, 2011.

13 KOUTSOURAS, G. W.; RAMOS, R. L.; MARTINEZ, L. R. Role of microglia in fungal infections of the central nervous system. Virulence, v. 8, n. 6, p. 705-7018, 2017.

14 STARKEY, J.; MORITANI, T.; KIRBY, P. MRI of CNS fungal infections: review of aspergillosis to histoplasmosis and everything in between. Clin Neuroradiol, v. 24, n. 3, p. 217-230, 2014.

15 ESHER, S. K.; ZARAGOZA, O.; ALSPAUGH, J. A. Cryptococcal pathogenic mechanisms: A dangerous trip from the environment to the brain. Mem Inst Oswaldo Cruz, v. 113, n. 7, p. e180057, 2018.

16 MCCARTHY, M. et al. Mold infections of the central nervous system. N Engl J Med, v. 371, n. 2, p. 150-160, 2014.

17 KANTARCIOGLU, A. S. et al. An updated comprehensive systematic review of Cladophialophora bantiana and analysis of epidemiology, clinical characteristics, and 
outcome of cerebral cases. Med Mycol, v. 55, n. 6, p. 579-604, 2017.

18 STOTT, K. E.; HOPE, W. Pharmacokinetics-pharmacodynamics of antifungal agents in the central nervous system. Expert Opin Drug Metab Toxicol, v. 14, n. 8, p. 803815, 2018.

19 MA, Y. et al. Central nervous system aspergillosis in immunocompetent patients: Case series and literature review. Medicine (Baltimore), v. 99, n. 44, p. e22911, 2020.

20 ULETT, K. B. et al. Cerebral cryptococcoma mimicking glioblastoma. BMJ Case Rep, v. 2017, 2017.

21 ZHANG, Y.; WU, X.; HU, Y. P. Aspergillosis infection over 20 years: A case report of probable vascular invasion in central nervous system. BMC Neurol, v. 20, n. 1, p. $342,2020$.

22 DE ALMEIDA, S. M. et al. Primary Central Nervous System Infection by Histoplasma in an Immunocompetent Adult. Mycopathologia, v. 185, n. 2, p. 331$338,2020$.

23 BARDE, F. et al. Low central nervous system posaconazole concentrations during cerebral phaeohyphomycosis. Antimicrob Agents Chemother, v. 63, n. 11, p. e0118419, 2019.

24 CHEN, Y. J. et al. Fusarium brain abscess in a patient with diabetes mellitus and liver cirrhosis. Acta Neurol Taiwan, v. 26, n. 3, p. 128-132, 2017.

25 SHUKLA, A. et al. Central nervous system mycosis: Analysis of 10 cases. Indian J Pathol Microbiol, v. 57, n. 4, p. 591-594, 2014.

26 GADGIL, N. et al. Curvularia brain abscess. J Clin Neurosci, v. 20, n. 1, p. 173-175, 2013.

27 DOYMAZ, M. Z. et al. A case of cerebral phaeohyphomycosis caused by Fonsecaea monophora, a neurotropic dematiaceous fungus, and a review of the literature. Mycoses, v. 58, n. 3, p. 187-192, 2015.

28 PUNIA, P. et al. Occupational CNS aspergillosis in an immunocompetent individual a diagnostic challange. Arch Environ Occup Health, v. 73, n. 6, p. 381-384, 2018.

29 VAN SANFORD, C. et al. A case of fatal invasive aspergillosis in a patient with neurosarcoidosis treated with infliximab. Int J Neurosci, v. 129, n. 6, p. 619-622, 2019. 
30 AKINS, P. T.; JIAN, B. The Frozen Brain State of Cryptococcus gattii: A GlobeTrotting, Tropical, Neurotropic Fungus. Neurocrit Care, v. 30, n. 2, p. 272-279, 2019.

31 MURAOKA, S. et al. Cerebral Infarction and Subarachnoid Hemorrhage Caused by Central Nervous System Aspergillus Infection. World Neurosurg, v. 90, p. 705.e9705.e13, 2016.

32 MLINARIĆ MISSONI, E.; BARŠIĆ, B. Two rare cases of central nervous system opportunistic mycoses. Arh Hig Rada Toksikol, v. 63, n. 4, p. 505-511, 2012.

33 TONE, K. et al. Epidural abscess caused by Schizophyllum commune: A rare case of rhinogenic cranial complication by a filamentous basidiomycete. Mycoses, v. 61, n. 3, p. 213-217, 2018.

34 PATEL, M. S. et al. Successful long-term management of invasive cerebral fungal infection following liver transplantation. Mycoses, v. 58, n. 3, p. 181-186, 2015.

35 BOURNE, E. L.; DIMOU, J. Invasive central nervous system aspergillosis in a patient with Crohn's disease after treatment with infliximab and corticosteroids. J Clin Neurosci, v. 30, p. 163-164, 2016.

36 XIAO, A. et al. Invasive intracranial aspergillosis spread by the pterygopalatine fossa in an immunocompetent patient. Braz J Infect Dis, v. 16, n. 2, p. 192-195, 2012.

37 RAY, S. et al. Special scenarios in the management of central nervous system aspergillosis: A case series and review of literature. Postgrad Med J, v. 95, n. 1125, p. $1-8,2019$.

38 FARROKH, S.; AVDIC, E. Voriconazole Autoinduction and Saturable Metabolism After Cessation of Rifampin in a Patient With Invasive Central Nervous System Aspergillus: Importance of Therapeutic Drug Monitoring. J Pharm Pract, v. 32, n. 5, p. 589-594, 2019.

39 KOURKOUMPETIS, T. K. et al. Central nervous system aspergillosis: A series of 14 cases from a general hospital and review of 123 cases from the literature. Medicine (Baltimore), v. 91, n. 6, p. 328-336, 2012.

40 ABID, M. B.; STROMICH, J.; GUNDACKER, N. D. Is ibrutinib associated with disseminated cryptococcosis with CNS involvement? Cancer Biol Ther, v. 20, n. 2, p. 138-140, 2019.

41 PAIVA, A. L. C. et al. Cryptococcoma mimicking a brain tumor in an 
immunocompetent patient: Case report of an extremely rare presentation. Sao Paulo Med J, v. 136, n. 5, p. 492-496, 2018.

42 MEARELLI, F. et al. Invasive filamentous fungus infection with secondary cerebral vasculitis in a patient with no obvious immune suppression. Int J Infect Dis, v. 19, p. 91-92, 2014.

43 KUMAR, D. et al. CNS aspergilloma mimicking tumors: Review of CNS aspergillus infection imaging characteristics in the immunocompetent population. J Neuroradiol, v. 45, n. 3, p. 169-176, 2018.

44 ROJAS-JAIMES, J.; CASTILlO CORDOVA, R.; TÁRRAGA GONZALES, D. Paracoccidiomicosis in the central nervous system: a case report. Rev Peru Med Exp Salud Publica, v. 32, n. 1, p. 10-12, 2015.

45 NOWAK, S. et al. Voriconazole as mono-therapy in orbitofrontal erosive aspergillosis without gross total resection: A case report and review of literature. Clin Neurol Neurosurg, v. 172, p. 93-95, 2018.

46 AL OTAIBI, F. E. Fatal case of cerebral aspergilloma complicated by ventriculitis and bacteremia due to Salmonella species in a sickle cell disease patient. Saudi Med J, v. 39, n. 9, p. 935-939, 2018.

47 VAIDYA, P. et al. A rare case report of aspergillosis of CNS presenting as a clival mass causing SAH from a mycotic aneurysm in an immunocompetent patient. Neurol India, v. 68, n. 1, p. 202-203, 2020.

48 NAKAMURA, Y. et al. Scedosporium aurantiacum brain abscess after near-drowning in a survivor of a tsunami in Japan. Respir Investig, v. 51, n. 4, p. 207-211, 2013.

49 KUAN, C. S. et al. Genomic analyses of Cladophialophora bantiana, a major cause of cerebral phaeohyphomycosis provides insight into its lifestyle, virulence and adaption in host. PLoS ONE, v. 11, n. 8, p. e0161008, 2016.

50 CAgGiano, G. et al. The Importance of a Proper Aetiological Diagnosis in the Management of Patients with Invasive Mycoses: A Case Report of a Brain Abscess by Scedosporium apiospermum. Mycopathologia, v. 172, n. 4, p. 317-322, 2011. 